\title{
Communication préventive et objectivation des risques : le cas des sports d'hiver
}

\section{Dominique Poizat}

\section{(2) OpenEdition}

12 Journals

Édition électronique

URL : http://journals.openedition.org/communicationorganisation/2561

DOI : 10.4000/communicationorganisation.2561

ISSN : $1775-3546$

\section{Éditeur}

Presses universitaires de Bordeaux

\section{Édition imprimée}

Date de publication : 1 novembre 2001

ISSN : 1168-5549

\section{Référence électronique}

Dominique Poizat, «Communication préventive et objectivation des risques : le cas des sports d'hiver », Communication et organisation [En ligne], 20 | 2001, mis en ligne le 27 mars 2012, consulté le 21 décembre 2020. URL : http://journals.openedition.org/communicationorganisation/2561 ; DOI : https://doi.org/10.4000/communicationorganisation.2561

Ce document a été généré automatiquement le 21 décembre 2020.

(c) Presses universitaires de Bordeaux 


\title{
Communication préventive et objectivation des risques : le cas des sports d'hiver
}

\author{
Dominique Poizat
}

\section{La thématique sécuritaire}

La gestion des risques constitue aujourd'hui un sujet de préoccupation majeur pour nos sociétés. Les discours récurrents annonçant une «montée de l'insécurité » ou encore l'exploitation politique de la thématique sécuritaire sont le reflet d'une société crispée sur l'impossible maîtrise d'une incertitude effective et symbolique qu'elle ne peut cesser de produire. Tous les champs d'activités sont aujourd'hui soumis à une prescription sécuritaire, qu'il s'agisse de notre environnement technologique ou naturel ou encore, de nos activités quotidiennes les plus triviales. Pourtant, l'aléa reste toujours présent; régulièrement, des événements accidentels nous rappellent l'impuissance de l'homme face aux phénomènes naturels ou relevant de l'activité humaine. Les médias s'en emparent le plus souvent en projetant systématiquement au devant de la scène les responsabilités individuelles ou collectives, la «culpabilité » d'un acteur ou d'un groupe d'acteur. Accepter la fatalité revient à reconnaître nos propres limites, aussi bien sur le plan technologique que d'un point de vue moral et ontologique; indirectement, la mise en scène médiatique de l'insécurité institue en retour la thématique sécuritaire en sujet de préoccupation majeure. «Le risque 0 n'existe pas!»; ce slogan, apparu dans le milieu des années 90, est aujourd'hui systématiquement associé à tout discours sur le risque. Il relève d'une trivialité elle-même porteuse de sens : serait-il possible que le risque 0 puisse exister et quel regard porter sur une société qui doit régulièrement rappeler à ses membres des truismes aussi élémentaires? Parallèlement au refus d'une fatalité déterministe, qui projette hors du champ conceptuel la dimension aléatoire, aussi réduite soit-elle, du libre arbitre, le risque acquière de plus en plus une réalité matérielle; il prend la forme unique d'un fait objectif et donc "objectivable», disposant d'une existence intrinsèque. Or, le risque «ne peut être extrait du contexte social dans lequel il 
survient. Son sens ne se construit que par rapport au fonctionnement d'une communauté humaine. Il n'a pas de substance abstraite, d'existence en soi $»^{1}$ (Decrop, Dourlens, VidalNaquet, 1997). Le risque ne peut être appréhendé seulement à travers une objectivation de ses référents, qui transitent nécessairement par un «contrat » entre l'individu et ses environnements. Notre objet est de mettre en évidence l'importance, d'un point de vue communicationnel, des rapports qui s'instaurent entre le subjectif et l'objectif, entre la prise en charge individuelle ou "internalisée » et collective ou "externalisée » de la gestion des risques, à partir du champ spécifique des activités ludiques et sportives de pleine nature, qui comprennent toutes les pratiques ayant pour cadre d'expression les espaces naturels propices aux activités libres et supposant un exercice individuel ou en groupes restreints. D'emblée, la notion de "pleine nature » pose problème car elle s'appuie sur la symbolique de l'aventure, de l'exploit, des grands espaces, de l'hygiène... dans un cadre authentique alors que la pleine nature est avant tout une nature " pleinement aménagée " pour le tourisme (sportif)-Résultant d'un besoin contemporain d'évasion, celui-ci en modifie la valeur d'usage et en fait le terrain privilégié de la redécouverte paradoxale d'une authenticité purement arte factuelle, construite selon les normes imposées par les développements économiques locaux et nationaux. Le besoin d'évasion dans une "nature sauvage " participe d'une forme de "dénégation du réel sur la base d'une appréhension avide et multipliée de ses signes"(Baudrillard, 1970), pour paraphraser Baudrillard. Il constitue l'essentiel du produit proposé aux utilisateurs des espaces montagnards. Cette approche démystificatrice de la nature permet de mettre en évidence le double mouvement de déterritorialisation-reterritorialisation des pratiques, c'est-à-dire, schématiquement, une déconstruction et une réappropriation de référents originels disposant d'une forte dimension symbolique réalisées sur la base d'une confrontation entre stratégies d'acteurs, qui se traduit par une mutation des représentations et des comportements. Les pratiques, qui, jusqu'au milieu du $\mathrm{XX}^{\mathrm{e}}$ siècle, s'ancraient sur l'apprentissage laborieux et progressif d'un milieu hostile, participent aujourd'hui d'un hédonisme fondé sur une volonté collective de jouissance immédiate. "La recherche d'un ajustement des pratiques aux possibilités et dispositions personnelles appelle un raccourcissement des temps d'initiation et un allégement des rituels d'apprentissage $»^{3}$ (Pociello, 1995). Les sports de pleine nature s'inscrivent dans une interdépendance entre deux paradoxes génériques: d'une part, toute forme de consommation touristique, fondée sur le mythe de la découverte des grands espaces naturels et sauvages, neutralise l'objet même de la consommation du simple fait d'un aménagement touristique qui "dénature " aussi bien les espaces que les pratiques; d'autre part, les pratiques en elles-mêmes s'appuient sur une banalisation du risque et simultanément, d'une dramatisation de l'accident. Pour Le Breton, "À l'absence de limites dans une société en perpétuel changement, réplique une recherche intime de limites de fait. Le contact symbolique avec la mort constitue l'une des voix les plus sollicitées pour se situer, se reconnaître un parmi les autres, trouver un goût de vivre qui se dérobe en partie. Une recherche sauvage de sens se joue là, dans le secret des recours individuels $»^{4}$ (Le Breton, 1996). Mais paradoxalement, l'aventure contemporaine dans une nature originelle fait appel à des technologies modernes : « Le paradoxe de la liberté est de ne jamais aller sans boussole pour s'éclairer $»^{5}$. De part leur ancienneté et compte tenu des processus de consommation de masse qu'ils génèrent, les sports d'hiver concentrent l'essentiel de la problématique sécuritaire des activités ludiques et sportives de pleine nature. Leur spécificité, en référence à la sécurité, porte sur la domination d'un marché qui a toujours défini les formes et les limites adoptées par la communication 
préventive. Celle-ci, par ailleurs, dépend d'organisations et d'acteurs qui, pour la plupart, sont étroitement impliqués dans la gestion économique des sports d'hiver. Notre questionnement porte donc sur la communication (publique) préventive dans un système de marché et les conclusions qui s'en dégagent ne peuvent systématiquement faire l'objet d'une inférence à d'autres domaines du risque.

\section{Un contexte global conflictuel}

2 Un premier travail réalisé en 1996 nous a permis de souligner un paradoxe fondamental propre à la communication préventive dans les sports d'hiver : celle-ci, envisagée, dans une perspective réductrice, sous l'angle idéologique de la solution "miracle » à une situation identifiée comme critique d'un point de vue sécuritaire, se situe, en fait, au centre des dysfonctionnements qu'elle est censée résoudre. Contrairement aux objectifs qui lui sont assignés, la communication préventive ne répond pas, en premier lieu, à une nécessité d'informer et de former les pratiquants sur les risques; au contraire, c'est l'information, considérée et présentée comme un impératif, qui devient, via la communication préventive, un outil permettant de diffuser des représentations et des valeurs concourant au développement de logiques parallèles divergentes et en contradiction avec une finalité préventive. Cette hypothèse suppose un élargissement de la problématique au-delà de la seule communication préventive afin d'analyser les interrelations entre les actions de prévention et de protection des risques en général et les logiques du système. Elle se situe en rupture avec le positionnement dominant des acteurs impliqués dans la gestion des risques, orienté vers une segmentation du champ des sports d'hiver ainsi qu'une disjonction de la problématique sécuritaire vis-à-vis de l'organisation globale du système. Ce positionnement se traduit, pour l'essentiel, par une centralisation des questions sécuritaires sur un nombre restreint d'acteurs qui conforte un repli corporatiste déjà très présent dans le milieu des professionnels de la montagne, et par un renforcement des paradoxes génériques dans le champ de la communication préventive des sports d'hiver. Brièvement, nous pouvons identifier les paradoxes aux différents niveaux : sociologique, économique, juridique, politique et médiatique, outre les paradoxes propres à toute forme de prévention qui résident, schématiquement, d'une part, dans le fait de devoir nommer l'objet dont la communication ajustement pour objectif de modifier ou de supprimer l'usage et d'autre part, dans l'ambivalence entre la sensibilisation, l'adhésion volontaire des individus et la dimension sous-jacente coercitive de la communication publique. La première forme paradoxale tient à un phénomène de société dont les sports d'hiver constituent le lieu privilégié d'expression. La sécurité est devenue une "nécessité sociale " alors que la thématique de l'aventure (Pociello), de l'extrême dans la quête d'un sens (Le Breton), d'une identification à des valeurs contre culturelles (Loret), donc indirectement, la prise de risques plus ou moins délibérée, devient elle-même socialement nécessaire et constitue une forme de régulation. L'action sportive est non seulement génératrice de risques mais qui plus est, le risque devient cosubstantiel à son organisation. Plus largement, la prise de risques est socialement valorisée, notamment dans le monde professionnel (Ehrenberg). L'acceptation du risque participe amplement, par ailleurs, à une rhétorique libérale ${ }^{6}$ prônant l'individualisation de la gestion des dysfonctionnements conséquents à l'organisation de la société, en opposition aux actions et mesures collectives sociales; d'où l'aspect sensible d'une approche socio-constructiviste $\mathrm{du}$ risque fondée sur la «responsabilisation» et 
l'« autonomisation » des pratiquants, compte tenu des « détournements libéraux » dont elle peut faire l'objet. Le second niveau s'exprime dans la confrontation d'une logique économique et d'une finalité préventive et la subordination de la seconde à la première. D'une part, la thématique sécuritaire, après avoir été considérée comme anticommerciale, donc proscrite, jusqu'à la fin des années 90, devient aujourd'hui un argument de vente, ce qui ne modifie guère les données du problème car en finalité, la communication préventive reste toujours " économiquement déterminée ». D’autre part, tous les supports de promotion, à commencer par les dépliants touristiques, valorisent indirectement les référents du risque et les réduisent à un simple produit de consommation. L'exploitation marketing d'un "extrême de masse" n'a fait que s'intensifier ces dernières années avec l'apparition et le développement du « free ride $»^{7}$. Une logique économique dominante s'accorde mal avec une finalité préventive qui remet en cause l'innocuité du produit « sports d'hiver ». La troisième forme de paradoxe se situe à un niveau juridique ; une "judiciarisation » des espaces de pratiques tend à s'imposer depuis le milieu des années 90 . Les orientations actuelles de la gestion des risques se déploient dans le sens d'une mise en place de plus en plus systématique de procédures visant à objectiver les référents du risque accompagnée d'une indifférenciation des compétences individuelles - alors qu'a contrario, les stratégies marketing ventent la personnalisation du produit -, au détriment d'une information indispensable à un engagement réfléchi, autonome et responsable du pratiquant face au risque. Le processus de judiciarisation des espaces et des pratiques et ses applications dépossèdent les individus de leurs pouvoirs de décision et progressivement les déresponsabilise. Le concept de « responsabilité » se trouve ainsi réduit à la dimension purement juridique d'un respect ou d'un refus des consignes de sécurité. D'un comportement " responsable " fondé sur les compétences individuelles, l'évolution actuelle s'oriente vers un comportement " discipliné » réduit au respect de "règles comportementales objectives ", alors que simultanément, l'objectivation des référents du risque est très limitée dans les milieux naturels et ne peut en aucun cas se substituer à une appréciation subjective ${ }^{8}$; c'est en cela que réside l'essentiel de l'ambiguïté. Cette forme de rationalisation, que nous préférons nommer, en rapport à notre objet « processus d'objectivation des référents du risque " pose problème dans le sens où elle institue des cadres de plus en plus formels à l'action sportive, et plus largement à l'action sociale définie comme « action humaine dans les différents milieux sociaux ${ }^{9}$ (Rocher, 1968), alors que les pratiquants, quelles que soient leurs modalités de pratique, sont avant tout motivés par un désir d'échapper aux cadres posés par la quotidienneté. Ainsi, les processus d'objectivation des référents du risque engendrent un recul permanent des limites de l'action, voire la recherche de nouvelles échappatoires, donc de nouvelles formes de prise de risques tout en favorisant simultanément un état de méconnaissance totale du milieu des sports d'hiver par les pratiquants. Le quatrième niveau de paradoxe réside dans les enjeux politique du développement économique local par le tourisme hivernal, confrontés aux responsabilités des maires qui ont à charge les pouvoirs de police sur leur territoire communal (et au-dessus d'eux, les préfets de départements). Si les compétences des maires peuvent faire l'objet d'une délégation (exemple : prise en charge de la sécurité des pistes, en général, par l'exploitant de remontées mécaniques), en revanche, en aucun cas leurs pouvoirs de police ne peuvent être délégués. Enfin, la dernière forme paradoxale que nous identifions se situe au niveau de la médiatisation omniprésente des sports d'hiver, qu'il s'agisse de celle des pratiques sportives dans la presse spécialisée, la télévision ou les supports vidéo, de la médiatisation des accidents ou de la médiatisation 
de type publicitaire. D'un côté, les médias en mettant en scène et en banalisant l'exploit sportif et plus largement, le champ de l'action sportive engagée, repoussent sans cesse les limites de l'« exceptionnel » et se font les vecteurs par excellence de représentations, de valeurs, et de processus d'identification fondés sur le risque. D'un autre côté, les accidents, lorsqu'ils surviennent, sont exploités dans une logique de l'immédiateté et du sensationnel qui ne laisse aucune place à une analyse critique de l'événement et qui génère la diffusion d'informations inadaptées, quand elles ne sont pas fausses, et orientées uniquement vers la culpabilisation des acteurs. Les effets des médias sur les représentations et les connaissances du public obéissent à des processus plus complexes qu'une simple relation de type cause à effet. Cependant, la diffusion d'informations contraires aux formes élémentaires des messages préventifs les plus adéquats, dans le cadre d'un événement dramatique produisant une forme de «traumatisme collectif, ne peut rester sans effet sur les représentations intégrées par les auditeurs et lecteurs pratiquants de sports d'hiver.

\section{Le système des sports d'hiver}

3 La communication préventive, produite à l'intérieur du système des sports d'hiver, se heurte donc à un ensemble de logiques contradictoires génératrices de dysfonctionnements. Or, ces dysfonctionnements sont structurels dans le sens où, non seulement ils s'inscrivent dans l'organisation du système mais qui plus est, ils participent et permettent cette organisation et de fait, sont "fonctionnels". La dimension dysfonctionnelle de certains phénomènes relève avant tout du point de vue de l'observateur; le comportement d'un acteur, qu'il s'agisse d'un pratiquant, d'un professionnel de l'encadrement ou d'un agent de la sécurité des pistes, dysfonctionnel selon un angle sécuritaire devient tout à fait fonctionnel s'il est observé d'un point de vue économique, ou encore juridique. De fait, la plupart des acteurs impliqués dans la gestion des risques sont déterminés par une pluralité de logiques divergentes très éloignées d'une finalité préventive; dans bien des cas, l'aspect préventif, donc central, de la communication sur le risque, passe au second plan des préoccupations majeures. Il est tout à fait courant, lors de réunions ayant pour objet un système d'information, que son efficacité informationnelle ne soit évoquée qu'en dernier lieu ou même totalement oubliée, après que soient abordées les questions économiques (ne pas effrayer la clientèle) et juridiques (le système n'engage-t-il pas excessivement la responsabilité des acteurs). Il nous est donc apparu essentiel de ne pas isoler la communication préventive et plus largement les actions de prévention et de protection des risques de l'organisation globale $\mathrm{du}$ système. Une approche constructiviste-systémique se présentait comme la mieux adaptée à notre terrain. De fait, il paraissait peu pertinent d'étudier les articulations entre la communication préventive et les logiques du système sans considérer, plus largement, le contexte informationnel dans lequel toute information de type préventif prend son sens; l'intérêt d'une étude sur la communication du risque ne porte pas, en premier lieu, sur les formes adoptées par la communication en elle-même, mais davantage sur ses modalités d'intégration dans le système et les contradictions qui en résultent. Nous observons plusieurs niveaux d'information. En premier lieu, les informations préventives et relatives à la sécurité qui concernent directement les actions de communication sur le risque auprès du public. En second lieu, les informations échangées entre acteurs dans le cadre de la gestion des risques. En troisième lieu, les 
informations indicielles, intersubjectives informelles et médiatisées qui relèvent, soit d'une émanation du milieu et des environnements des sports d'hiver et supposent un rapport étroit entre le signifiant et le signifié, soit des relations entre les individus, soit de la médiatisation des sports d'hiver. En dernier lieu, les informations «hypercontextuelles ", qui participent à un niveau sociétal élargi de l'éducation aux risques et «citoyenne » et de la construction sociale du risque. Notre méthode s'appuie sur une enquête ${ }^{10}$ auprès des pratiquants dans l'objectif d'évaluer leurs connaissances, leurs représentations, leurs comportements et leurs opinions sur le milieu des sports d'hiver, complétée par des entretiens semi-directifs et par les apports d'une observation participative de l'organisation de la gestion des risques dans le cadre d'une activité professionnelle. Aucune étude de ce type n'avait été réalisée auparavant dans ce champ. L'analyse adopte le point de vue du récepteur des messages préventifs dans un premier temps, pour s'élargir dans un second temps aux logiques déterminantes du système et aux stratégies d'acteurs. Dans le cadre d'une problématique communicationelle, nous avons choisi de ne pas recentrer le questionnement sur un aspect spécifique, tel que la médiatisation des sports d'hiver, qui n'aurait pu prendre en compte les interrelations entre les différents processus à l'intérieur du système et les mécanismes dysfonctionnels résultant d'une confrontation entre des logiques contradictoires structurelles.

\section{Les processus d'objectivation des risques}

Les rapports entre la prise en charge externalisée et la gestion subjective du risque, et donc les processus d'objectivation du risque et leurs conséquences, constituent un aspect central de la communication préventive. Une première définition, inspirée des risques majeurs naturels et technologiques, présente le risque comme la conjonction de l'« aléa » ${ }^{11}$ et des « enjeux $»^{12}$ ou $~ "$ le croisement de l'aléa et de la vulnérabilité $»^{13}$ (Coste, 2001). D'emblée, cette définition axée sur une problématique de gestion collective du risque ne satisfait pas à un domaine pour lequel la décision en amont de l'action accorde une place prépondérante i la subjectivité de l'individu. Quel que soit le type de risque considéré, dès lors que celui-ci est considéré en fonction des facteurs humains qui le déterminent, il doit impérativement intégrer la dimension comportementale des individus, que ne laisse pas suffisamment percevoir les notions d'« enjeu » ou de "vulnérabilité ». Le risque résulte du rapport triangulaire " aléas-enjeux-comportements », d'autant plus prégnant lorsque la décision et l'action humaines sont déterminantes en amont de l'événement (accidentel). Cette première approche introduit la dimension centrale des représentations dans la construction sociale du risque. "En tout état de cause, le risque est une construction sociale $»^{14}$ (Roqueplo, 1997). Appréhender le risque selon une approche constructiviste permet d'opérer la distinction fondamentale entre ce qui relève d'une probabilité événementielle objective et partiellement « objectivable » de ce qui relève des relations subjectives de l'individu à son environnement. Toutefois, une problématique sécuritaire a ceci de singulier qu'elle nous confronte particulièrement aux limites du constructivisme et nous recentre donc, nécessairement, sur la double perspective d'une réalité objective "de premier ordre " et d'une réalité construite "de second ordre » ${ }^{15}$ (Watzlawick, 1978). Le risque participe d'une construction sociale et simultanément, procède d'une dimension phénoménale objective, et c'est à travers le jeu des relations qui se créent entre les processus objectifs et subjectifs que se détermine une gestion globale des risques. Or, les évolutions actuelles tendent vers des processus systématiques 
d'objectivation des risques, c'est-à-dire vers une régulation de l'action en fonction de normes objectives introduites par des référents collectifs, reconnus par tous, comme uniques principes d'aide à la décision et d'évaluation des droits, devoirs et responsabilités des acteurs. Il est possible d'identifier trois orientations principales dans les processus d'objectivation des risques. La première orientation provient des évolutions juridiques et implique une objectivation directe des référents du risque. Afin de définir les responsabilités des acteurs, suite à un accident, les magistrats recherchent, à travers les différents éléments d'appréciation disponibles, des référents objectifs les moins sujets à interprétation. Mais ces référents n'existent pratiquement pas en montagne. Les réglementations se limitent à un arrêté municipal type succinct et, depuis quelques années seulement, à des arrêtés municipaux et préfectoraux ponctuels lors d'une situation nivo-météorologique exceptionnelle. La définition des espaces sécurisés et des domaines hors-pistes, quant à elle, fait l'objet d'une ambiguïté totale; rien ne délimite actuellement avec précision les espaces de pratiques selon des zones de compétences et de responsabilités en fonction des acteurs. Les jurisprudences conséquentes aux accidents de ces dernières années s'appuient en grande partie sur les systèmes d'informations préventives et relatives à la sécurité et sur les systèmes de protection pour établir les responsabilités des acteurs mis en cause. Dès lors, nous assistons à un détournement d'usage des tous les systèmes existants (drapeau d'avalanche; échelle européenne du risque d'avalanche; bulletins météorologiques... mais aussi les systèmes de protection individuels tels que l'Arva ${ }^{16} \ldots$..). Ces détournements d'usage constituent des processus d'objectivation. D'une part, ils imposent à l'ensemble des actions de communication une autre destination que celle initialement prévue; la communication du risque et plus largement tous les moyens mis au service de la prévention et de la protection deviennent ainsi définis en fonction d'une finalité juridique et non préventive. D'autre part, outre le fait qu'ils supposent une fiabilité totale des moyens matériels et humains d'estimation des risques qui sont loin de constituer une "science exacte", ils transforment des informations relatives aux comportements "subjectifs" à adopter de l'ordre de la recommandation, en norme comportementale à respecter de l'ordre de l'interdiction. La seconde orientation concerne le champ économique et se traduit par une objectivation indirecte du risque via une production de formes de consommation génératrices de prises de risques. Affichant une ambiance générale de détente, mais fortement connotés par l'action, l'aventure, la performance, la confrontation, les supports publicitaires mettent en exergue les pratiques sportives hivernales à travers la cénesthésie des conduites de vertige. L'aventure dans une nature vierge, la recherche de sensations, d'émotions, la confrontation aux limites individuelles, dont l'expression peut se résumer par la tendance " free ride " actuelle, sont les composants d'un idéal de performance ramené à l'état de produit marchand pour une consommation de masse. Le processus d'objectivation relève ici en premier lieu d'une objectivation de l'action, le principe consistant à réduire symboliquement la distance entre, d'un côté, ce qui est " possible » et, d'un autre côté, ce que l'insuffisance en compétences techniques, physiques et cognitives subjectives ne permet pas. Les espaces d'aventures sont désormais présentés comme accessibles à tous, noyés dans des contenus textuels et iconographiques qui mêlent sans distinction des registres hétéroclites (" détente en famille », « soirées raclette au coin du feu », « sauts de barres rocheuses » et autres sensations extrêmes). En finalité, objectivation de l'action et objectivation du risque ne modifient guère la teneur d'une problématique sécuritaire et se rejoignent dans un processus global d'objectivation; compte tenu de la dépendance entre l'action et la prise de risques, une objectivation de la première affecte 
nécessairement la seconde. La troisième orientation des processus d'objectivation se rapporte à la médiatisation des sports d'hiver et plus spécifiquement à celle des accidents. La recherche systématique de responsabilités associée à l'inexactitude et à l'inadéquation des informations diffusées dans le cadre du traitement médiatique de l'événement, renforce et coproduit le détournement d'usage des systèmes d'informations opéré dans le champ juridique. La présentation qui a été donnée du drapeau à damier, à travers la médiatisation du drame de la crête du Lauzet du 23 janvier 1998, laissant place à une interprétation du type "hors-pistes interdit» alors qu'il signifie "risque localisé d'avalanche", illustre tout à fait nos propos. Ce type de médiatisation accentue la responsabilité du guide auprès de l'opinion publique et simultanément, véhicule la représentation d'un pouvoir de décision réduit au seul respect d'un interdit qui se substitue d'office à une recommandation originelle. Aucune place n'est laissée à l'erreur humaine d'appréciation d'un ensemble de facteurs; tout accident est perçu en tant qu'événement prévisible et tout risque devient, symboliquement, totalement maitrisable dès lors que les pratiquants, amateurs et professionnels confondus, respectent les informations «objectives indiscutables» et se situent dans une prétendue norme comportementale «responsable». L'objectivation médiatique du risque abstrait totalement la dimension subjective centrale dans la gestion des risques et génère ainsi des représentations et des comportements inadaptés aux réalités environnementales.

\section{Conclusion}

5 La communication du risque dans le domaine des sports d'hiver, déterminée par un ensemble de logiques divergentes et en contradiction totale avec une finalité de prévention et d'éducation aux risques, arrive difficilement à trouver ses marques. La centralisation des questions sécuritaires, jusqu'aux milieux des années 90, autour d'un nombre restreint d'acteurs, encore fortement présente aujourd'hui, se traduit par un repli corporatiste alors que la diversité et l'hétérogénéité des processus en action supposerait une approche transversale du problème par les acteurs impliqués dans la gestion des risques, dans un esprit de concertation et de collaboration concrétisé autour de groupes de travail. Mais cette centralisation s'inscrit plus largement dans une forme d'opacité propre à la gestion des risques dans un système de marché pour lequel toute information concernant de près ou de loin la thématique sécuritaire est " particulièrement sensible ", du fait qu'elle se réfère nécessairement à des éléments dont l'évocation ne s'accorde guère avec la promotion des produits constitués par les sports d'hiver. L'information préventive, paradoxalement, reste aujourd'hui emprunte d'une forte valeur anti-commerciale alors que la sécurité est de plus en plus appréhendée par les stations comme un gage de qualité. Il ne faut pas "effrayer le client» tout en lui montrant habilement que des actions collectives sont réalisées en vue d'une sécurité maximum. Globalement, la gestion des risques s'inscrit donc dans des stratégies morcelées résultant d'un questionnement superficiel qui ne s'appuie sur aucune méthode rigoureuse de recueil de données. De fait, il n'existe pas de politique globale, fondée sur des objectifs clairement définis, pour la simple raison que ces politiques supposeraient nécessairement d'aborder les dysfonctionnements actuels en profondeur, c'est-à-dire en observant de quelles manières ils servent l'organisation du système, notamment selon les orientations économiques et juridiques, ce qui ne satisfait pas les stratégies de nombreux acteurs parallèlement impliqués dans la gestion des risques et dans l'économie des sports 
d'hiver. Une telle situation favorise la mise en place de procédures et plus généralement d'une objectivation des référents du risque. Ce processus est d'autant plus insidieux et prééminent qu'il s'inscrit dans une forme d'« auto contrôle », c'est-à-dire informel, et procède par ajustements successifs des modalités d'actions, en fonction de la croissance des responsabilités juridiques. Ces ajustements relèvent principalement d'un ensemble d'adaptations au " coup par coup ", hétérogènes et disparates. Le détournement des outils de communication sur le risque et plus largement de prévention et de protection, tout d'abord «juridique", mais aussi à des fins "économiques» et dans un contexte de médiatisation omniprésente, conforte et produit même les conditions d'une adoption par les pratiquants de comportements à risques auxquels la communication est censée apporter une réponse. Ce processus renforce l'inadéquation des représentations sur la montagne, sur les sports d'hiver et sur les risques inhérents aux pratiques. Plus généralement, il corrobore une représentation sociale du risque étendue à l'ensemble des activités humaines qui abstrait la dimension subjective centrale de sa gestion au profit d'une gestion collective « objectivée ». Il conforte ainsi la confusion déjà très prégnante, chez les individus, entre ce qui relève d'une logique coercitive réduisant le pouvoir de décision dans l'action à un simple comportement discipliné et ce qui participe d'une attitude globale responsable et autonome indissociable de l'engagement individuel dans des pratiques à risque. Le pratiquant de sport d'hiver devient un "client» dont les modalités de pratiques sont prises en charge "collectivement». Les résultats de notre enquête montrent une très faible implication des pratiquants dans la gestion de la sécurité. Un ensemble de corrélations confirme, d'une part, la pertinence d'un élargissement de la notion de "communication préventive " à celle d'« éducation aux risques ", et, d'autre part, la nécessité, dans une perspective de responsabilisation des individus, souvent évoquée mais rarement définie, de produire, en aval d'une éducation aux risques, des systèmes d'information pouvant être utiles et utilisés par des individus « responsables » et " autonomes », ce qui suppose une certaine transparence et une levée des ambiguïtés et des dysfonctionnements qui rendent actuellement ces systèmes inopérants. Plus précisément, un constat s'impose: plus les individus sont potentiellement en mesure de gérer subjectivement les risques, plus ils sont critiques visà-vis de ce qui se rapporte à la sécurité en général, et moins ils respectent les comportements définis par des règles et des normes collectives objectives. Outre la difficulté de produire des informations utilisables selon les différents niveaux de compétence des pratiquants, compte tenu de leur hétérogénéité, le paradoxe majeur réside dans le fait que les dysfonctionnements participent à l'organisation d'un système, dont la prévention n'est qu'un élément, ce qui nous permet de comprendre pourquoi, dans bien des cas, les actions de prévention et de protection, à l'inverse des objectifs qui leurs sont assignés, produisent en partie les processus qu'elles ont pour mission de solutionner. Dès lors, les actions de communication et de prévention ne peuvent être réellement efficaces si celles-ci, en amont, ne font pas l'objet d'un questionnement approfondi prenant en compte l'ensemble des logiques qui les déterminent et des paradoxes qui résultent des interrelations entre les processus en action. 


\section{BIBLIOGRAPHIE}

BAUDRILLARD, J., La société de consommation. Paris : Éditions Denoël, 1970.

DECROP, G., DOURLENS, C, VIDAL-NAQUET, P. A., Les scènes locales du risque. Institut National du Génie Urbain de Lyon, Pôle Grenoblois d'étude et de Recherche pour la Prévention des Risques Naturels, Institut d'étude de la Sécurité Civile, EDF, avec la collaboration de Denis Cœur (Ehra) et Olivier Marco (Cemagref), août 1997. LE BRETON, D., Passions du risque. Paris : Éditions Métailiè, 1996. POCIELLO, C, Les cultures sportives. Paris : PUF, 1995. POIZAT, D., Hors-pistes :

comportements, connaissances, et perceptions des pratiquants. In Neige et Avalanches, $\mathrm{n}^{\circ} 89$, mars 2000. ROCHER, G., L'action sociale. Paris : éditions HMH, Ltée, 1968.

ROQUEPLO, P., Entre savoir et décision, l'expertise scientifique. INRA, Paris, 1997.

WATZLAWICK, P., La réalité de la réalité. Paris : Le Seuil, 1978.

\section{NOTES}

1. Geneviève Decrop, Christine Dourler Pierre A. Vidal-Naquet, Les scènes locales du risque, août 1997, p. 19.

2. Jean Baudrillard, La société de consommation, Éditions Denoël, Paris, 1970, p. 33.

3. Christian Pociello, Les cultures sportives, PUF, Paris, 1995, p. 258.

4. David Le Breton, Passions du risque, Éditions Métailié, Paris, 1996, p. 162.

5. David Le Breton, ibid. p. 12.

6. Cf. l'article de Robert Castel, « «Risquophiles» et «risquophobes»: l'individu selon le Medef », in Le Monde du jeudi 7 juin 2001, p. 1 et p. 16.

7. Pratiques « libres » et « extrêmes».

8. Contrairement à un domaine comme celui de la circulation routière ou l'appréciation subjective reste présente mais réduite par de nombreux critères objectifs.

9. Guy Rocher, L'action sociale, éditions HMH, Ltée, Paris, 1968, p. 18.

10. Enquête réalisée durant la saison 1998-1999 auprès de 1221 pratiquants consultés sur les domaines aménagés et 1462 pratiquants par le biais d'une publication dans la revue Montagne Info $\mathrm{du}$ Club Alpin Français. Pour plus de détails sur la méthode et les premiers résultats, cf Dominique Poizat, «Hors-pistes: comportements, connaissances, et perceptions des pratiquants ", in Neige et Avalanches n 89, Mars 2000.

11. Probabilité pour qu'un événement se produise.

12. Tout ce qui peut être, en quelque sorte, soumis au risque considéré et, en retour, en modifier les caractéristiques (présence d'individus, infrastructures...).

13. Lucien Coste, "Sur plusieurs définitions du risque, et leurs conséquences en matière de prévision, prédiction et prévention » in La Houille Blanche, $\mathrm{n}^{\circ}$ 2, 2001, p. 18.

14. Philippe Roqueplo, Entre savoir et décision, l'expertise scientifique, INRA, Paris, 1997, p. 80.

15. Paul Watzlawick, La réalité de la réalité, Le Seuil, Paris, 1978, p. 138.

16. Appareil de Recherche aux Victimes en Avalanche. Dans une affaire récente, la possession d'un ARVA par un pratiquant à été considérée comme preuve que ce pratiquant été informé des risques d'avalanche, donc circonstance aggravante. Sans suite a postériori, mais il y a bel et bien eu tentative! 


\section{RÉSUMÉS}

Les actions de communication préventive sont aujourd'hui au centre des préoccupations des acteurs impliqués dans la gestion des risques dans le domaine des sports d'hiver. Mais ces actions s'inscrivent dans un « contexte informationnel global conflictuel» et se heurtent à un ensemble de logiques contradictoires génératrices de dysfonctionnements. Au centre des dysfonctionnements, les "processus d'objectivation des risques» constituent les orientations majeures de ces dernières années et ont inévitablement des effets sur la gestion des risques.

The actions of preventive communication are today in the center of the preoccupations of the actors involved in the winter sport safety management. But these actions are in keeping in a "global conflictury informationel context» and are camind up against contradictory process that product dysfunctions. In the center of these dysfunction, the "process of risk objectivation » are making up the main axes of these last years and have necessary effects on the risk management.

\section{INDEX}

Mots-clés : sports d'hiver, communication préventive, processus d'objectivation du risque, paradoxes, dysfonctionnements

\section{AUTEUR}

\section{DOMINIQUE POIZAT}

Dominique POIZAT, 44 ans ; doctorant au GRESEC (Groupe de Recherche Sur les Enjeux de la Communication) laboratoire de recherche de l'UFR de communication Grenoble III, Université Stendhal, Institut de la Communication et des Médias ; chargé d'étude à l'ANENA (Association Nationale pour l'Étude de la Neige et des Avalanches) à Grenoble ; ex-pistcur-secouriste dans les stations de l'Alpe du Grand Serre et de l'Alpe d'Huez. 